Article

\title{
Real-Time Temperature Estimation of Three-Core Medium-Voltage Cable Joint Based on Support Vector Regression
}

\author{
Jiangjun Ruan, Qinghua Zhan, Liezheng Tang * and Ke Tang \\ School of Electrical Engineering, Wuhan University, Wuhan 430072, China; ruan308@126.com (J.R.); \\ qhzhan@163.com (Q.Z.); Tangk205@126.com (K.T.) \\ * Correspondence: tlz9302@163.com; Tel.: +86-159-7200-6323
}

Received: 3 May 2018; Accepted: 28 May 2018; Published: 31 May 2018

\begin{abstract}
The joint is the weakest link in three-core medium-voltage power cable systems and the temperature is an essential indicator to its insulation condition. Therefore, a model to estimate the temperature inside the three-core cable joint was built based on support vector regression (SVR) with two fixed cable surface temperatures as inputs. The samples for model training were obtained from 3-D transient thermal analyses through finite element method (FEM) under different single-step currents. A temperature-rise test of $15 \mathrm{kV}$ three-core cable joint was carried out and the estimated temperature based on SVR agrees well with the measured result with a maximum error of about $4{ }^{\circ} \mathrm{C}$. Besides, the proposed model could accurately estimate the joint temperature even though the thermal conductivity of armor wrap used in thermal analysis for model training differs from its real value. The accuracies and calculation speed of the proposed model were compared with those of FEM, showing a better generality of our model. A temperature-rise test under unbalanced three-phase currents was performed and the temperature estimation errors are within $6{ }^{\circ} \mathrm{C}$, indicating the applicability of the method. The effect of contact resistance was briefly discussed in the end. This approach helps improve cable operation and maintenance.
\end{abstract}

Keywords: three-core medium-voltage cable joint; temperature monitoring; support vector regression; transient thermal analysis; temperature-rise test

\section{Introduction}

A large number of three-core medium-voltage power cables are widely used in the urban distribution network. According to the fault statistics of power cables in Jiangxi Province, China [1], the accidents of $10 \mathrm{kV}$ cables accounted for nearly $96 \%$ of accidents among the medium and high voltage cables. Since most of $10 \mathrm{kV}$ cables are three-core cables, the condition monitoring of three-core cables is considered essential to reduce failures. It is generally accepted that the joint is one of the weakest links of the power cable circuit [2-4]. Moreover, due to the thicker insulation layers and contact resistance, the cable joint usually represents the hot spot in the cable system $[5,6]$. With the rapid growth in the demand for electric power in urban areas, the cable load currents will greatly increase, thus making the overheating in joints more serious. The overheating together with high electric field will more probably lead to a breakdown or even explosion of cable joints. Therefore, it is really of significant practical value to monitor the temperature in three-core cable joints.

The research on thermal performance of the three-core cable have received much attention for many years. The calculation method for the conductor temperature in three-core cable primarily includes the analytical method [7,8], FEM [9-11] and the artificial intelligence algorithm [12].

But as for joints, although there has been much related research regarding single-core cable joints [3,13-15], little attention has been paid on the temperature monitoring in three-core cable joints. 
The relatively low reliability requirements and infrequent overload conditions of medium-voltage three-core cables may cause the little focus. But with the growing importance of distribution grid and the increasing electricity consumption in urban areas, the temperature monitoring of three-core cable joints will be particularly significant. Besides, people might think that the joint was not the hot spot in cable system without considering contact resistance in the past, which would be discussed later in discussion part. In the study on thermal analysis of three-core cable joints, Weedy B.M. [16,17] used a finite difference method to compute both the steady-state and transient temperature of a $33 \mathrm{kV}$ three-core cable and splice buried in the ground. The system was reduced to a 2-D field problem by replacing three-core cable of the same conductor section but with thicker insulation which gave the same conductor temperature. Nevertheless, the contact resistance was not considered and the algorithm was not verified through the temperature-rise test. Xu et al. [18] applied 2-D FEM to calculate the steady-state temperature field of $10 \mathrm{kV}$ three-core cable joint but did not consider the axial heat flux in the cable joint. Wang et al. [6] employed 3-D FEM to compute the steady-state temperature profile in three-core cable joint but the length of adjacent cable was not considered, which has a remarkable effect on the joint temperature. Gouda et al. [5] presented an analytical method to calculate the steady-state temperature along the joint of three-core cable and the calculated results were compared with those obtained by experimental study and the FEM.

To achieve real-time temperature monitoring of the three-core cable joint, the transient temperature rise calculation must be considered. Unfortunately, this problem remains unsolved according to the previous study introduced above. One way to overcome this issue is to carry out transient thermal analysis of three-core cable joint through 3-D FEM, but it is time-consuming to make this an impractical option for application in real-time monitoring.

In a cable joint where the radial as well as axial heat flow takes place, the temperature in cable adjacent to the joint will be affected by the joint. In contrast, the surface temperature of joint can hardly reflect the inner temperature in joint. Therefore, this paper puts forward a temperature calculation model for three-core cable joint based on support vector regression (SVR) with two fixed cable surface temperatures as inputs. The performance of this model was verified by temperature-rise test of $15 \mathrm{kV}$ three-core cable joint and discussed in detail.

\section{Principle of Temperature Estimation of Three-Core Cable Joint}

When a cable circuit with joint is overheated by the Joule heat of the conductor, both the conductor and the surface in the cable system will have a temperature rise in different extent. The surface temperatures in cable remote from joint and adjacent to joint reflect the thermal effect of cable and joint, respectively, and the combination of two factors can fully describe the temperature rise in joint. Hence, it is likely to estimate three-core cable joint temperature from cable surface temperatures of two spots, among which the one is close to joint and the other is remote from joint.

Accordingly, the key point of this method is to find out the relationship between the temperature of three-core cable joint and the two cable surface temperatures. It is similar to data mining, where the samples and the algorithm are crucial.

The samples can be obtained from various 3-D transient FEM thermal analyses under different single-step currents over the range considered in practice.

For algorithm, SVR, a machine learning algorithm developed from the optimal hyperplane in linear separable cases, is adopted to build the relationship. Since it is based upon the structural risk minimization principle and minimizes the empirical risk and confidence interval simultaneously to ensure the generalization [19], SVR is likely to estimate temperature of three-core cable joint.

\section{The 3-D Thermal Analysis of Three-Core Cable Joint}

In order to acquire the samples mentioned above, it is significant to conduct the thermal analysis of three-core cable joint. 


\subsection{The Structure}

The structure of $15 \mathrm{kV}$ three-core cable and joint is shown in Figure 1.

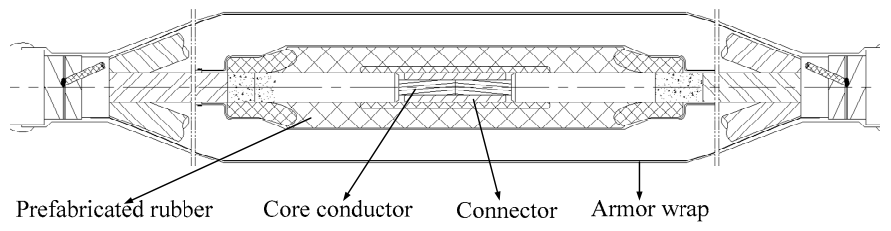

(a)

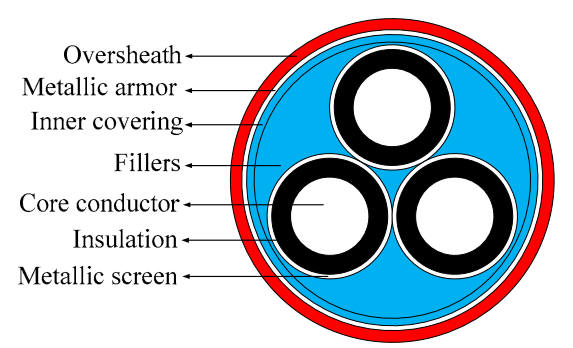

(b)

Figure 1. The schematic diagram of the three-core cable and joint. (a) Cable joint; (b) Cable.

\subsection{The Calculation Model}

Since the cable joint structure of three-core cable is complex and not axisymmetric, a 3-D model must be employed to accurately calculate the temperature profile of three-core cable joint. The model, illustrated in Figure 2, contains the joint and the surrounding cable long enough for longitudinal heat flow in the end to be zero.

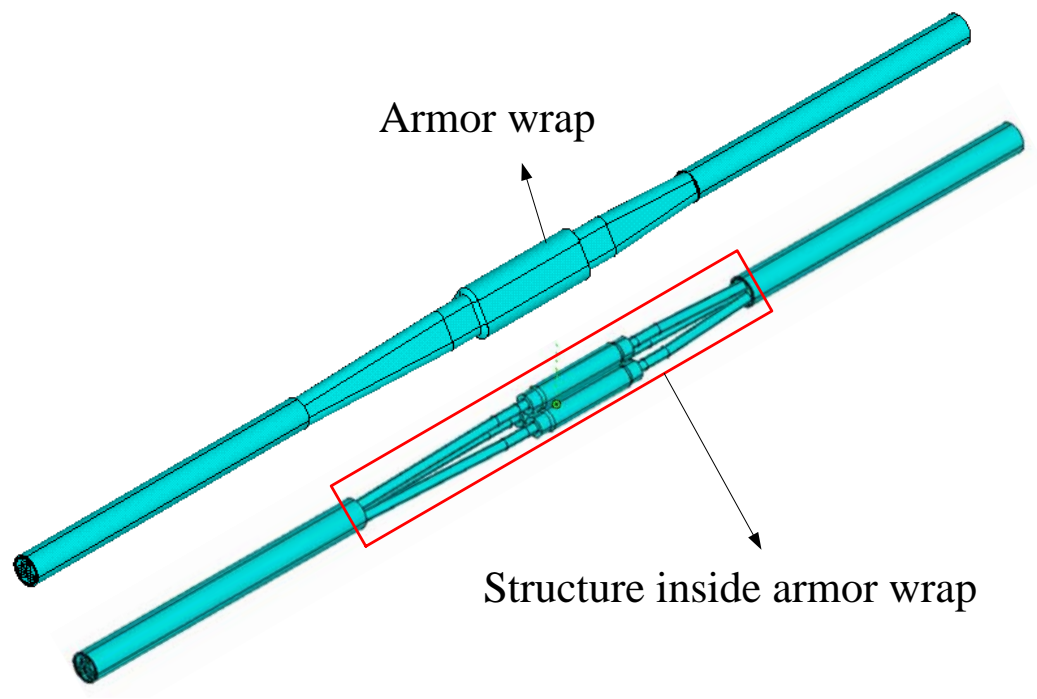

Figure 2. The calculation model of three-core cable joint.

\subsection{The Mesh Generation}

Because the shape of three-core cable joint is extremely irregular, if one kind of meshing is adopted for all regions, the number of elements will be too large to be calculated. In order to reduce the elements, different kinds of meshing were used for different regions according to their shapes.

The cable has a quite good repeatability along its axial direction. Therefore, the crosssection of the cable body was firstly meshed with quadrilateral-shaped elements which were then swept along the axial direction to form the hexahedral elements for cable, as depicted in Figure 3a. 


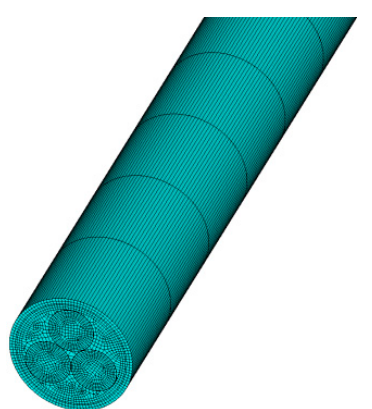

(a)

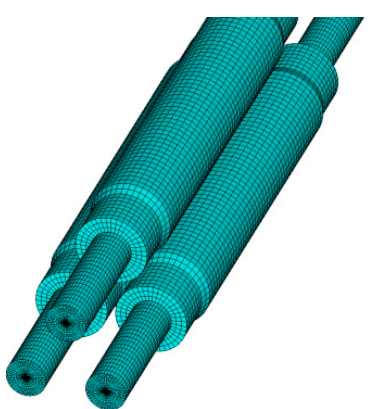

(b)

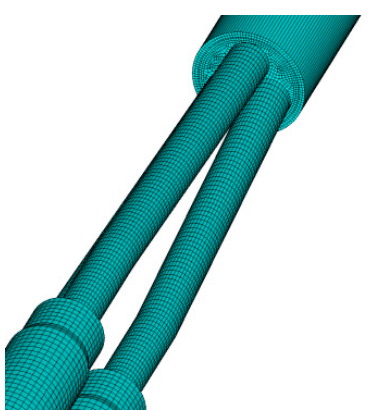

(c)

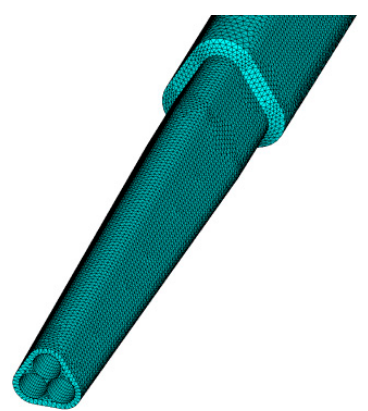

(d)

Figure 3. Mesh of each part in the three-core cable joint. (a) Cable; (b) Joint; (c) Connection region between cable and joint; (d) Air and armor wrap.

According to the axial symmetry of the joint, the 2-D axisymmetric section of joint in a phase was meshed with quadrilateral-shaped elements, and these 2-D elements were rotated about the axis to generate the 3-D hexahedral elements, as illustrated in Figure $3 \mathrm{~b}$.

After the mesh generation of cable and joint, the connection region between the cable and joint was meshed through sweeping 2-D elements from end of cable or joint, as shown in Figure 3c.

As for the armor wrap and inner air, the tetrahedral elements were created over the entire body through free meshing due to their irregular shape, as illustrated in Figure 3d.

Therefore, by employing the mesh sweeping and rotating technique, the number of nodes and elements is much smaller than ones meshed with the free mesher. In addition, the time to create these elements and for calculation is much smaller.

However, since the element shapes of the cable and joint are different, the elements of connection region differ from that of cable or joint. Hence, an approach to tie together two regions with dissimilar mesh patterns should be adopted.

One way to solve this problem is to generate constraint equations that connect the selected nodes of one region to the selected elements of the other region. As seen in Figure 4, at the interface between the two regions, nodes should be selected from the more dense mesh region, $\mathrm{A}$, while the elements should be selected from the less dense mesh region, B. The degrees of freedom in region A nodes are interpolated with the corresponding degrees of freedom of the nodes on the region $B$ elements, using the shape functions of the region B elements.

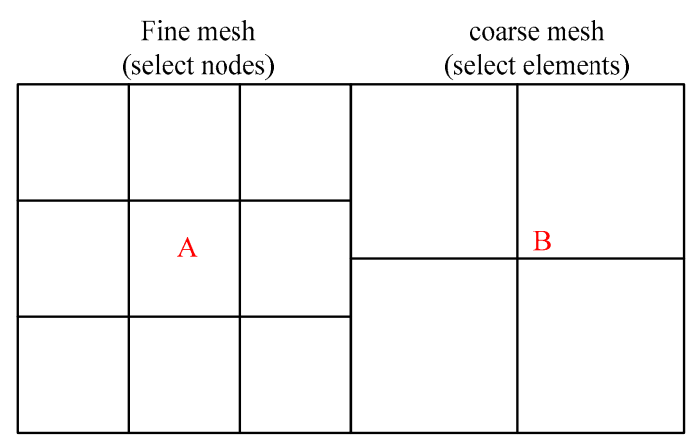

Figure 4. Schematic diagram of mesh coupling.

\subsection{Loss Calculation}

It is reasonable to neglect the losses in armor, dielectric and metallic screen, and only consider the conductor losses in a medium-voltage three-core cable. The conductor losses can be regarded as two 
parts, of which the one, $G_{1}$, comes from the core cable and the other, $G_{2}$, comes from the connector in the joint, as depicted in Figure 5. The losses can be calculated as follows.

$$
\begin{gathered}
G_{1}=\frac{I^{2} R_{1}}{V}=I^{2} \frac{\rho l_{1}}{S^{2} l_{1}}=I^{2} \frac{\rho}{\pi^{2} r^{4}} \\
G_{2}=\frac{W_{2}}{V}=\frac{I^{2} R}{\pi r_{1}{ }^{2} l_{2}}
\end{gathered}
$$

where $\rho$ is the electric resistivity of conductor; $l_{1}$ and $l_{2}$ are the length of conductor and connector, respectively; $r$ and $r_{1}$ are the radius of conductor and connector, respectively; $R$ is the equivalent resistance of the connector and its internal conductor, taken as $7.5 \mu \Omega$ in this paper, which was measured through Micro-Ohm Meter after the compression of connectors was finished during the assembly of cable joint.

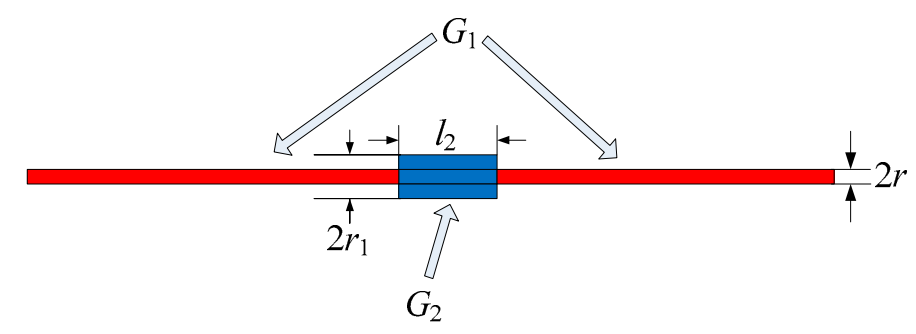

Figure 5. Schematic diagram of conductor losses in three-core joint considering contact resistance.

In this paper, the skineffect was not taken into consideration because the skineffect only increases the total losses to cause a linearly increasing temperature rise but does not change the temperature distribution. Thus, the skineffect can be simply regarded as an increase of current in a certain proportion at $0 \mathrm{~Hz}$. Since the currents in simulation is over the range considered in practice, an increase of current in a certain proportion will have a minor effect on the temperature relationship between cable joint and cable surfaces. Thus, our model based on SVR remains almost unchanged and unaffected by the skineffect.

\subsection{Boundary Condition Settings}

Since the cable is long enough for longitudinal heat flux in the end to be zero, the truncation surfaces of two cables in both sides satisfy the boundary condition of the second kind, where the normal gradient of temperature is zero. When the temperature $T$ is given on the surface $S$, the boundary condition reads

$$
\left.\frac{\partial T}{\partial n}\right|_{S}=0
$$

In the thermal analysis, the cable and joint are assumed to be surrounded by air. Thus, the surfaces of both cable and joint satisfy the boundary condition of the third kind. Let $T_{a a}, \lambda$ and $h$ respectively be the ambient temperature, the thermal conductivity of surface and the heat transfer coefficient between surface and air. The boundary condition of the third kind takes the form

$$
\left.\lambda \frac{\partial T}{\partial n}\right|_{S}=h\left(T_{a}-T\right)
$$

In the following simulations, the ambient temperature and heat transfer coefficient are all specified to $25^{\circ} \mathrm{C}$ and $8 \mathrm{~W} /\left(\mathrm{m}^{2} \cdot{ }^{\circ} \mathrm{C}\right)$, respectively. 


\subsection{The Steady-State Temperature Profile}

The steady-state temperature distributions of three-core cable joint with the current of $500 \mathrm{~A}$ are illustrated in Figure 6. It can be seen that cable surface is hotter than joint surface due to the thicker insulation and the hot spot is located in the connector inside the joint.

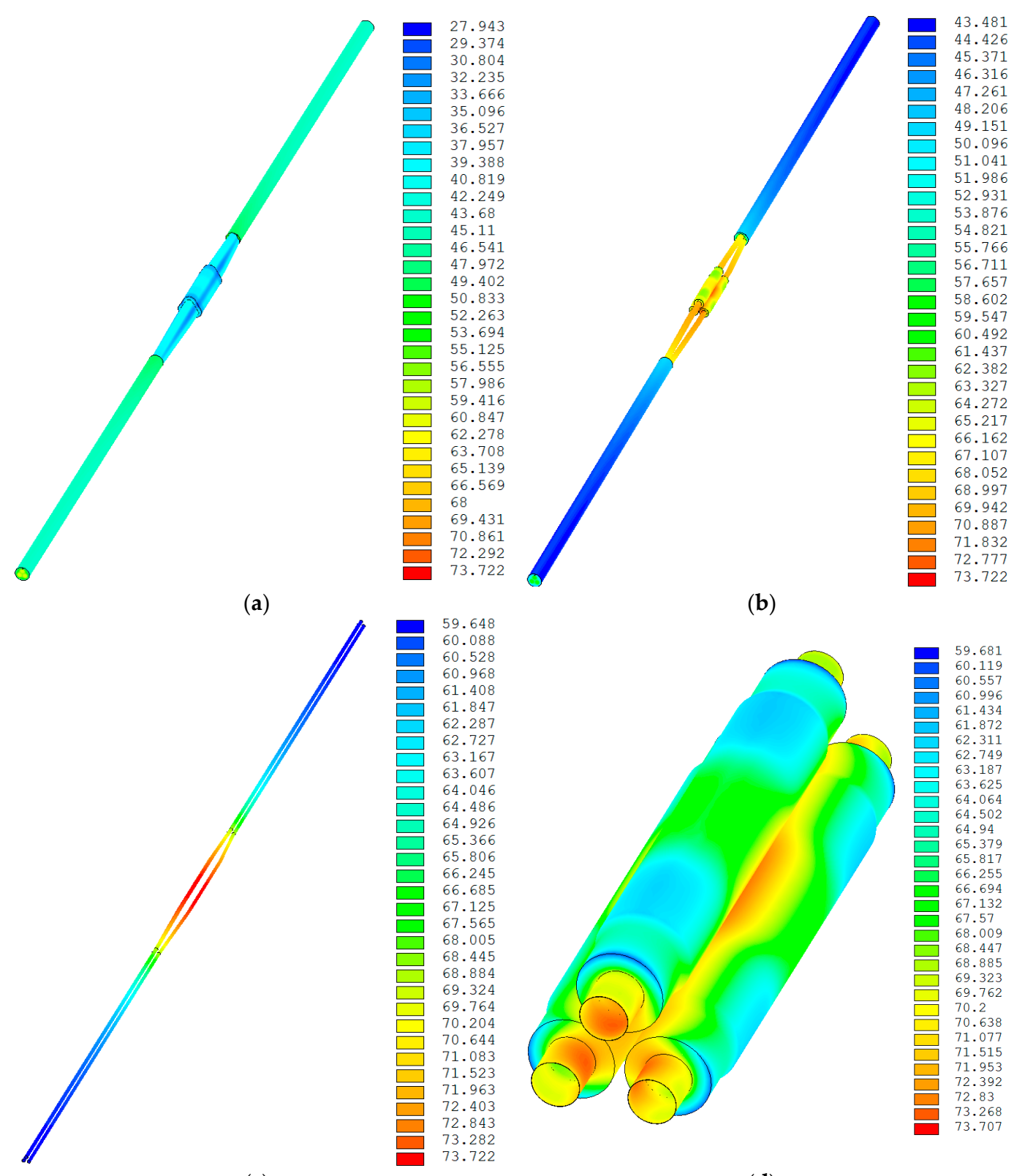

(c)

(d)

Figure 6. Steady-state temperature profile of three-core cable joint. (a) The entire body; (b) The body without armor wrap and air; (c) Core conductor; (d) Prefabricated rubber.

\subsection{The Transient Temperature Profile}

The transient temperature rise under variable loading calculated through FEM are illustrated in Figure 7, where spot 1 and spot 2 mean the surfaces of cable $0.25 \mathrm{~m}$ and $2.25 \mathrm{~m}$ away from the end of joint, respectively. From this figure, there is the same trend of temperature change inside the joint and on the cable surface under variable loading, which indicates their strong correlation. Therefore, the temperature inside the joint can be well estimated from the cable surface temperature in theory. 


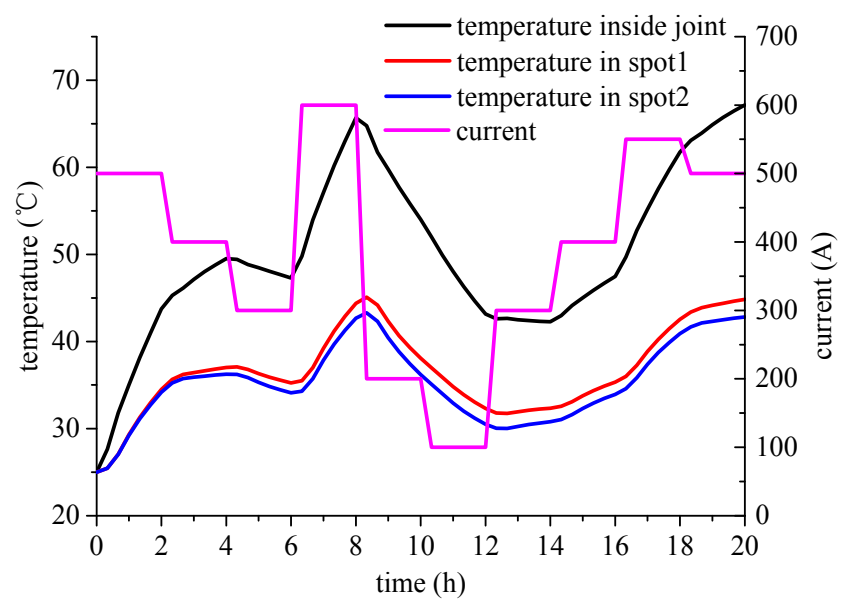

Figure 7. Transient temperature rise inside the joint and on the cable surface with variable loading.

\section{Model Optimization for Temperature Estimation in Three-Core Cable Joint}

\subsection{Parameter Selection of SVR}

The effectiveness of SVR depends upon the selection of kernel, the parameters of kernel, and soft margin parameter $C$. A common choice is a Gaussian kernel, which has a single parameter $\gamma$. The best combination of $C$ and $\gamma$ is often selected by a grid search with exponentially growing sequences of $C$ and $\gamma$, for example, $C \in\left\{2^{-5}, 2^{-3}, \ldots, 2^{13}, 2^{15}\right\} ; \gamma \in\left\{2^{-15}, 2^{-13}, \ldots, 2^{1}, 2^{3}\right\}$. Typically, each combination of parameter choices is checked using $v$-fold cross-validation, and the parameters with best cross-validation accuracy are picked.

In this paper, the maximum and minimum of $C$ in the search range were set to $2^{9}$ and $2^{13}$, respectively; the maximum and minimum of $\gamma$ in the search range are taken as $2^{0}$ and $2^{3}$. The search step is assumed to be $2^{0.1}$ for both $C$ and $\gamma$, and the 3 -fold crossvalidation is adopted.

\subsection{Determination of Number and Location of the Measuring Spots in Cable Surface}

For determination of the measuring spots in cable surface, three fixed points were selected for analysis. The distance of these points from end of the joint are $0.25 \mathrm{~m}, 0.75 \mathrm{~m}$, and $2.25 \mathrm{~m}$, as illustrated in Figure 8, and the temperatures in the corresponding spots are named $T_{1}, T_{2}$, and $T_{3}$, respectively. These temperatures are nearly an arithmetic progression to represent the surface temperature profile of three-core cable joint.

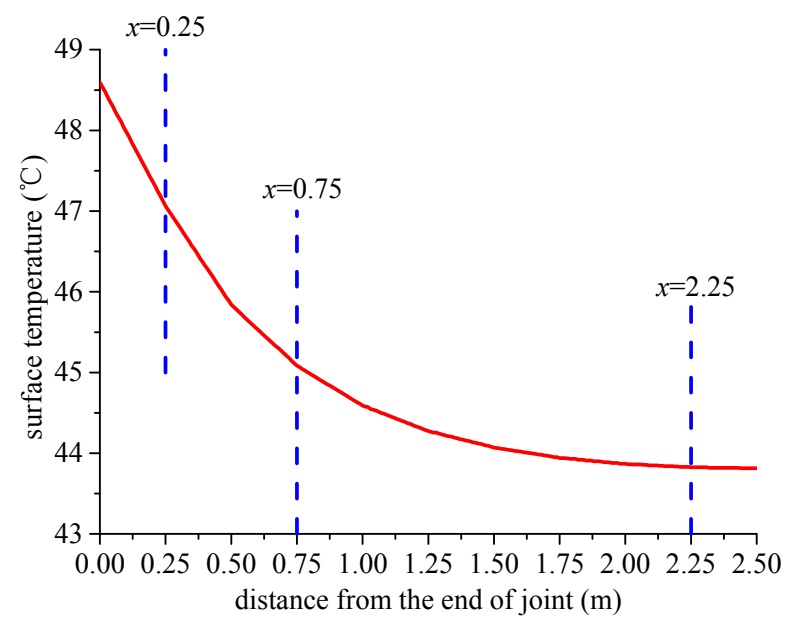

Figure 8. Schematic diagram of cable surface temperature in different measuring spots. 
The current rating of the three-core cable joint analyzed in this paper is about $500 \mathrm{~A}$. Considering a certain margin, the transient thermal analyses of cable joint were carried out under various constant currents ranging from $100 \mathrm{~A}$ to $600 \mathrm{~A}$ with the interval of $100 \mathrm{~A}$. Each simulation lasted $20 \mathrm{~h}$ with the data sampling period of $20 \mathrm{~min}$. The temperature $T_{0}$ inside the joint and cable surface temperatures were taken out as the output and inputs of training sample data, respectively. According to these data and parameter selection method described above, the temperature calculation model for three-core cable joint was established.

To verify the feasibility of the algorithm, a transient thermal analysis of three-core cable joint under variable current was performed and the cable surface temperatures obtained from this simulation were extracted as inputs of the proposed model. Compared with the cable joint temperatures simulated by FEM, the estimated temperature errors based on SVR with different measuring spots combination are shown in Table 1. It can be seen that when only one cable surface temperature is employed to estimate the temperature of joint, the error is largest and increases with the increasing distance from the joint. In the case of two cable surface temperatures, the optimal location is that one spot is close to the joint and the other is far from the joint. Moreover, the estimated error from three measuring spots is quite similar to that from two spots. Thus, the two cable surface temperatures, $T_{1}$ and $T_{3}$, were selected for temperature estimation in the following.

Table 1. Estimated temperature errors based on support vector regression (SVR) with different measuring spots combination.

\begin{tabular}{ccc}
\hline Measuring Spots & Root Mean Square Errors $\left({ }^{\circ} \mathbf{C}\right)$ & Maximum Errors $\left({ }^{\circ} \mathbf{C}\right)$ \\
\hline$T_{1}$ & 1.825 & 5.138 \\
$T_{2}$ & 2.472 & 5.773 \\
$T_{3}$ & 3.119 & 6.370 \\
$\left(T_{1}, T_{2}\right)$ & 1.134 & 4.023 \\
$\left(T_{1}, T_{3}\right)$ & 1.090 & 4.107 \\
$\left(T_{2}, T_{3}\right)$ & 1.428 & 4.453 \\
$\left(T_{1}, T_{2}, T_{3}\right)$ & 1.091 & 4.102 \\
\hline
\end{tabular}

\section{Temperature-Rise Test of Three-Core Cable Joint}

To validate the effectiveness of this model, the temperature-rise test for $15 \mathrm{kV}$ three-core cable joint was carried out. The test platform is illustrated in Figure 9 and the total length of the cable circuit is over $20 \mathrm{~m}$. Two measuring spots in the cable surface was fixed $0.25 \mathrm{~m}$ and $2.25 \mathrm{~m}$ distant away from the end of joint, respectively, according to previous analysis.

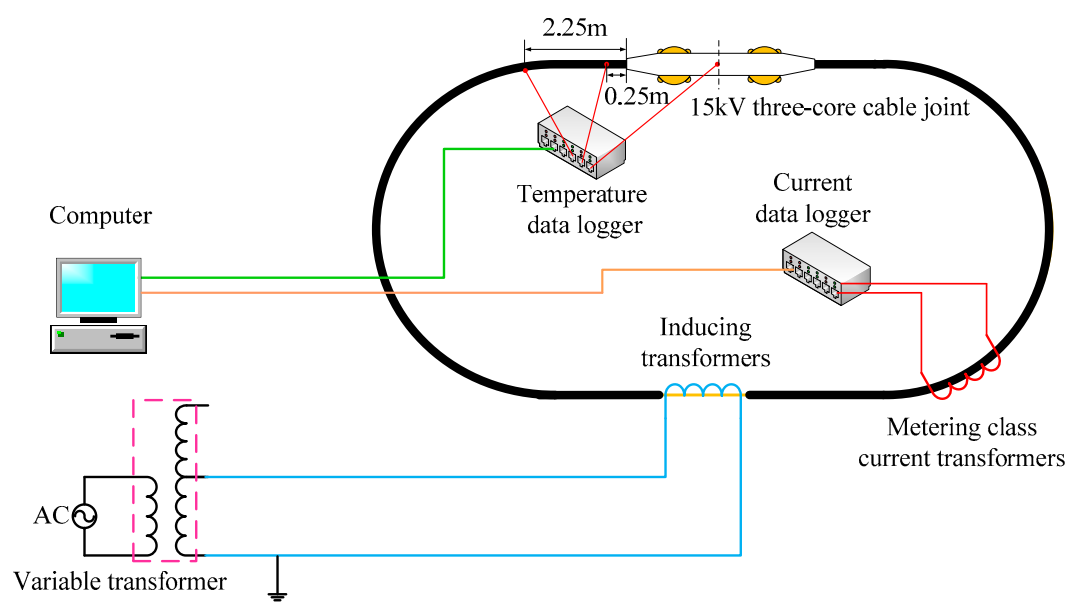

Figure 9. Schematic diagram of the temperature rise test platform. 
Distribution three-core cable is often with armor made of steel. When AC current is applied to the cable, there is a magnetic field in its armor together with the steel loss. If the current is three-phase symmetric, magnetic field is small enough to ignore the loss while if the cable is fed with a single-phase current, magnetic flux is large, thus causing a significant loss in the armor. Hence, a three-phase current was applied to the cable circuit during the temperature-rise test. The three-phase inducing transformers, as shown in Figure 10, were connected to the three-phase variable transformers to adjust the currents, which were measured through the metering class current transformers.

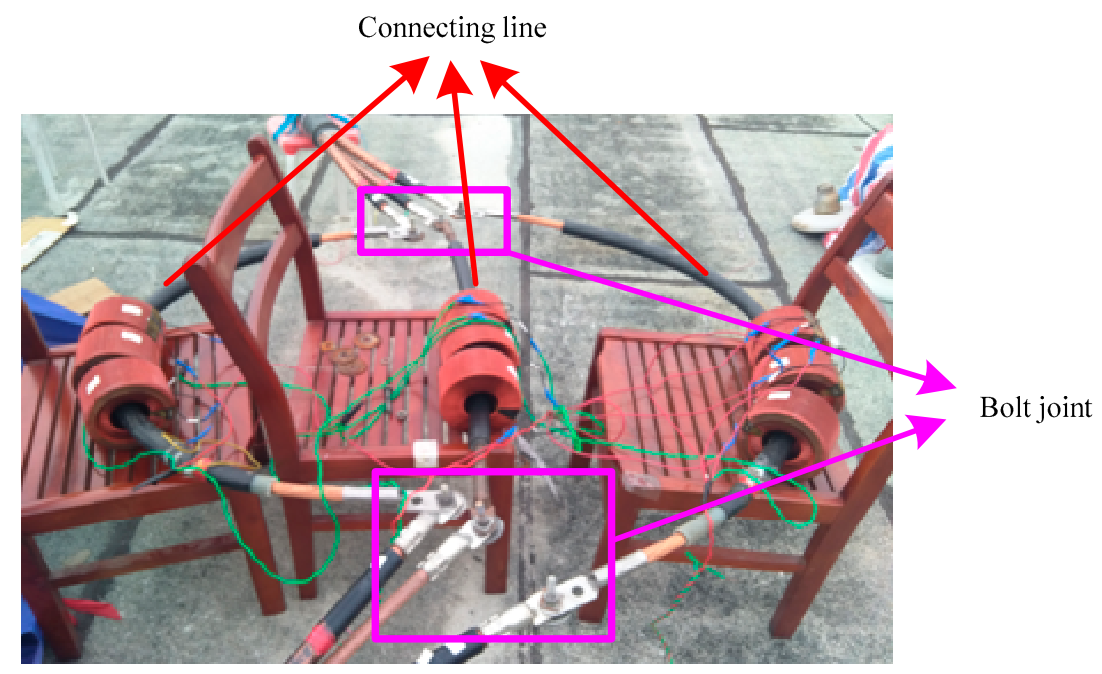

Figure 10. Photo of three-phase inducing transformers.

Platinum temperature sensors with tolerance class of A [20] were employed to measure the temperature, which could be monitored by the computer through temperature data logger. Since the circuit was not energized, thermometers were installed at the connector surface of the cable joint. As for the measuring spots in the cable, four thermometers were evenly attached on the circumference of the cable surface. The configuration of the three-core cable joint temperature-rise test is sketched in Figure 11.

The test lasted three days and two nights for $46 \mathrm{~h}$. The three-phase load currents, the temperature inside the cable joint, the cable surface temperatures as well as the ambient temperature were recorded every $1 \mathrm{~min}$.

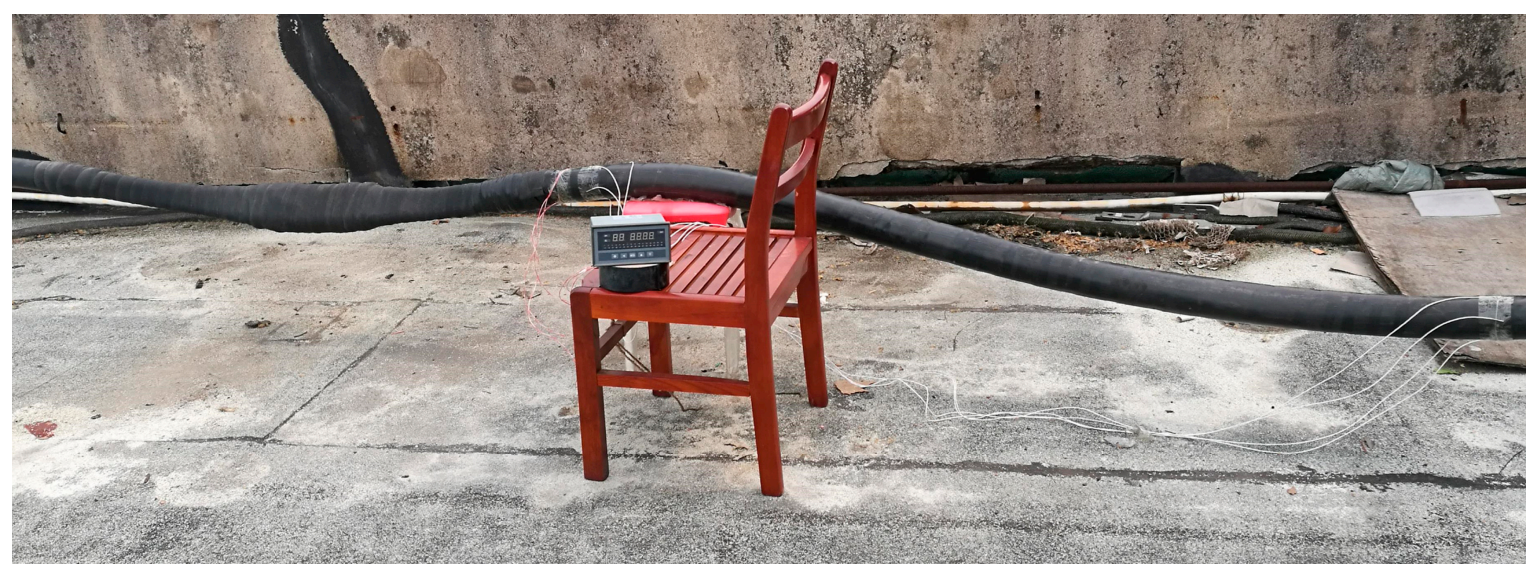

Figure 11. Configuration of the temperature-rise test. 


\section{Results and Discussions}

\subsection{Temperature Estimation of Three-Core Cable Joint Based on SVR}

The measured temperatures, as well as currents and the estimated temperatures based on SVR with the cable surface temperatures in two fixed points as inputs, are illustrated in Figure 12. Due to the different lengths of the connecting lines and different contact resistances of bolt joints, as seen in Figure 10, the impedances of three loops are not the same. For this reason, although the voltages applied to these inducing transformers were three-phase symmetric, the induced currents were unbalanced, even with a maximum difference of almost 100 A. Even though three-phase currents injection resulted in 100 A difference, the single-phase current injection would cause a much larger loss in armor. This is because that the net current giving rise to steel loss is the same as the single-phase current which can reach $500 \mathrm{~A}$, thus making the test totally different from the engineering practice. On the other hand, the power system allows unbalanced currents with maximum unbalanced degree of $25 \%$, and 100 A current difference corresponds to about unbalanced degree of $20 \%$. Therefore, the armor loss caused by unbalanced three-phase currents during our test is within the range of the loss in the actual power grid.

The measured temperature inside the cable joint is the average temperature of the three-phase conductors. During the test, the ambient temperature changed slowly between $19.4{ }^{\circ} \mathrm{C}$ and $27.8^{\circ} \mathrm{C}$.

It can be seen from the figure that the estimated temperature is in good agreement with the measured result with the maximum error of approximately $4{ }^{\circ} \mathrm{C}$ under the variable three-phase currents. In addition, the temperature errors increased with a sudden change of the current. This is because there is a time delay of the temperature change between the cable surface and the inside conductor. Nevertheless, the errors were within the acceptable range in engineering application.

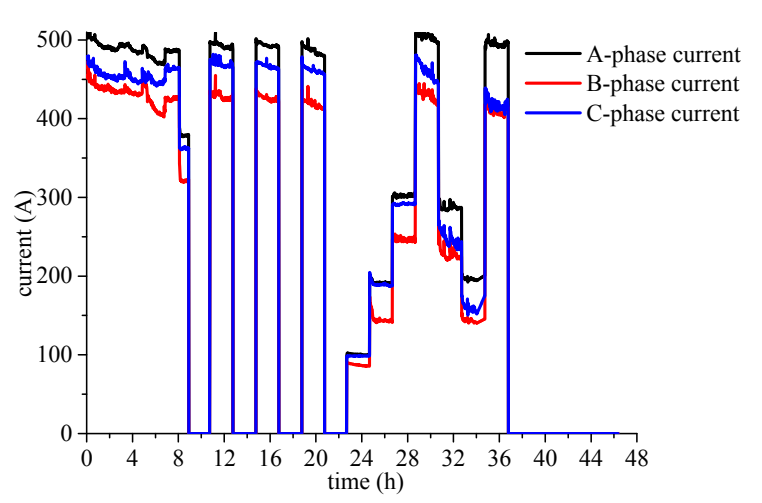

(a)

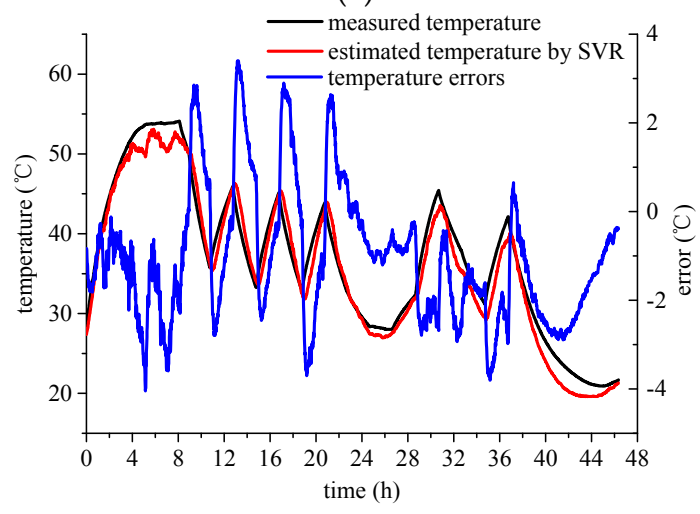

(b)

Figure 12. The measured results and estimated temperature based on SVR. (a) Current; (b) Temperatures inside the three-core cable joint. 


\subsection{The Effect of Thermal Conductivity in Armor Wrap on Temperature Estimation}

The thermal parameters of cable and prefabricated rubber are stable and recommended by IEC standards [21]. However, the thermal conductivity $\lambda_{\mathrm{a}}$ of armor wrap is usually uncertain because of its porous structure. Since the armor wrap is made from fiberglass, the thermal conductivity $\lambda_{\mathrm{a}}$ of armor wrap was set to $0.04 \mathrm{~W} /(\mathrm{m} \cdot \mathrm{K})$, the same as that of fiberglass [22], in this paper. However, the thermal conductivity $\lambda_{\mathrm{a}}$ of armor wrap was assumed to be $0.14 \mathrm{~W} /(\mathrm{m} \cdot \mathrm{K})$ by Wang [6]. Thus, it is necessary to analyze the effect of thermal conductivity in armor wrap on temperature estimation.

For the first $8 \mathrm{~h}$ during the temperature-rise test, the three-phase current kept almost constant at $487.8 \mathrm{~A}, 431.5 \mathrm{~A}$, and $455.3 \mathrm{~A}$ and the temperature inside the cable joint reached steady gradually. To determine the real value of thermal conductivity in the armor wrap is close to $0.04 \mathrm{~W} /(\mathrm{m} \cdot \mathrm{K})$ or $0.14 \mathrm{~W} /(\mathrm{m} \cdot \mathrm{K})$, a steady-state FEM thermal analysis of three-core cable joint with different thermal conductivity in armor wrap was conducted. The current and ambient temperature used for simulation were the same as the that of test temperatures.

The measured temperatures inside cable joint and the estimated results based on SVR are shown in Table 2. It can be seen that the thermal conductivity $\lambda_{\mathrm{a}}$ in armor wrap is much more close to $0.14 \mathrm{~W} /(\mathrm{m} \cdot \mathrm{K})$ than to $0.04 \mathrm{~W} /(\mathrm{m} \cdot \mathrm{K})$. However, the model of temperature estimation was established from the sample data of the thermal analysis where $\lambda_{\mathrm{a}}=0.04 \mathrm{~W} /(\mathrm{m} \cdot \mathrm{K})$. This results indicate that although the thermal conductivity of armor wrap used in thermal analysis for model training differs from its real value, the proposed model are still suitable for the temperature estimation inside cable joint, and this is quite useful in the engineering application.

Table 2. Comparison between measured temperatures inside cable joint and estimated results.

\begin{tabular}{cccc}
\hline Phase & $\begin{array}{c}\text { Measured } \\
\text { Temperatures }\left({ }^{\circ} \mathrm{C}\right)\end{array}$ & $\begin{array}{c}\text { Estimated } \\
\text { Temperatures }\left({ }^{\circ} \mathbf{C}\right) \text { with } \\
\lambda_{\mathbf{a}}=\mathbf{0 . 1 4} \mathbf{~ W} /(\mathbf{m} \cdot \mathbf{K})\end{array}$ & $\begin{array}{c}\text { Estimated } \\
\text { Temperatures }\left({ }^{\circ} \mathbf{C}\right) \text { with } \\
\lambda_{\mathbf{a}}=\mathbf{0 . 0 4} \mathbf{~ W} /(\mathbf{m} \cdot \mathbf{K})\end{array}$ \\
\hline$A$ & 55.70 & 58.85 & 69.45 \\
$B$ & 53.20 & 55.27 & 65.28 \\
$C$ & 53.30 & 56.73 & 66.96 \\
\hline
\end{tabular}

\subsection{The Accuracies Comparison between the Proposed Method and Other Methods}

According to the literature search mentioned above, there are totally three kinds of methods to calculate the temperature of three-core cable joint, including FEM, finite difference method $[16,17]$ and analytical method [5]. But the analytical method can only solve steady-state problem so that it is hard to realize temperature monitoring.

Finite different method is essentially a thermal network where thermal resistances and capacitances are analogous to their electrical namesakes. Determination of thermal resistances with irregular shapes is the key point. In literature [16,17], the irregular thermal resistances, as seen in Figure 13, were calculated using the so-called "G-factors", which can be obtained by 2-D dimensional field plots in IEC 60287 [23].

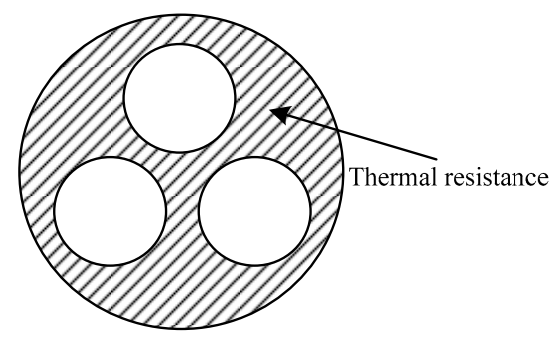

Figure 13. The irregular thermal resistance in literature [16,17]. 
The model of joint in this paper, however, is not the same as the above one. The crosssection of three-core cable joint is shown in Figure 14. It can be seen that the outer surface of joint is not a circle but a chamfered triangle. Thus, "G-factors" can not be used to calculate this part of thermal resistance. What's more, a very important assumption made in $[16,17]$ was that the cable and joint outer surfaces are isothermal. Nevertheless, according to the FEM thermal analysis as illustrated in Figure 15, the temperature difference in outer surface of the joint can reach over $10{ }^{\circ} \mathrm{C}$ due to its triangular shape, which demonstrates the joint outer surface is not isothermal. Therefore, joint outer surface can not be regarded as a temperature node so that the finite difference method is hard to be applied to solve this temperature distribution.

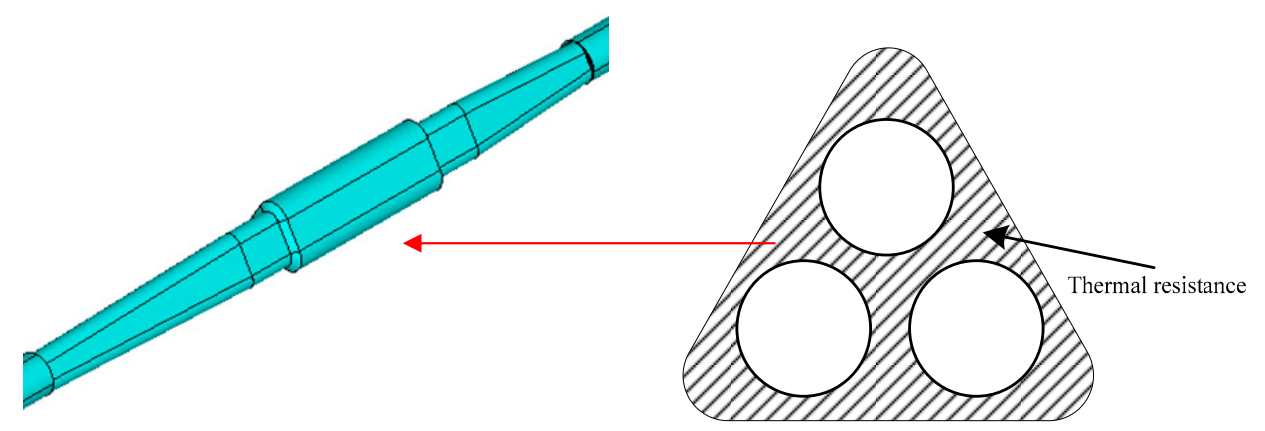

Figure 14. The model and irregular thermal resistance in this paper.

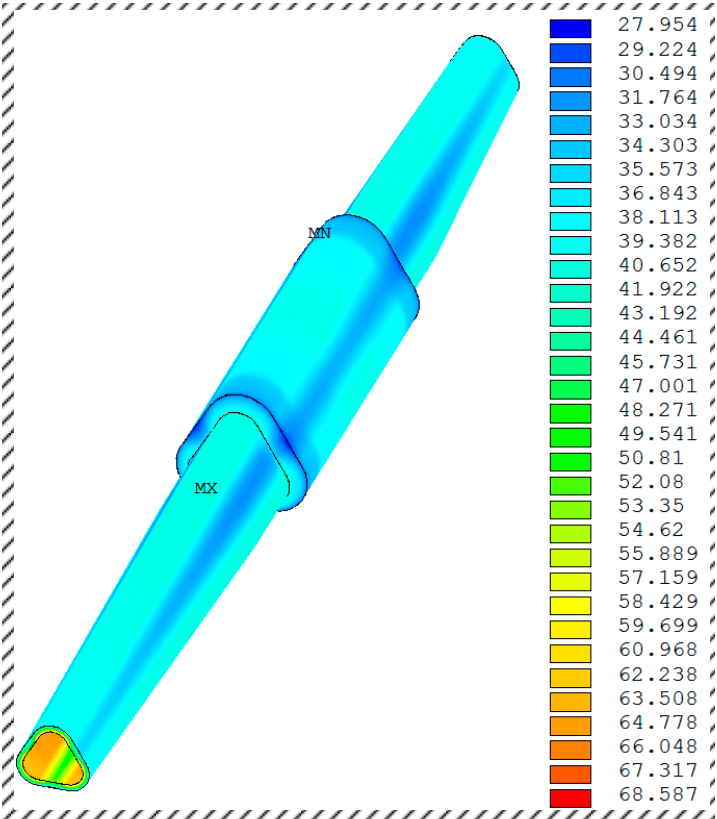

Figure 15. The temperature profile of armor wrap using finite element method (FEM) thermal analysis.

In the following, we would compare the accuracies of the proposed model with those of FEM model. The inputs of the proposed model were two surface temperatures obtained in the test and those of FEM were ambient temperature and load currents also recorded in the test. Both of the calculated temperatures were compared with measured temperature inside the cable joint for precision evaluation. The calculated temperature by FEM is the average temperature of the three-phase conductors due to the acceptable unbalanced degree of currents.

Since the thermal conductivity of armor wrap is sometimes uncertain, the following analyses were carried out in two cases. Firstly, suppose that the thermal conductivity $\lambda_{\mathrm{a}}$ of armor wrap in thermal analysis was $0.14 \mathrm{~W} /(\mathrm{m} \cdot \mathrm{K})$. The temperature errors of the two methods are shown in Figure 16. It can 
be seen that both of the two models have high accuracies. The mean absolute deviations of FEM and SVM model are 1.65 and $1.71{ }^{\circ} \mathrm{C}$, respectively, which indicates that the accuracies of FEM are a bit higher than that of SVM.

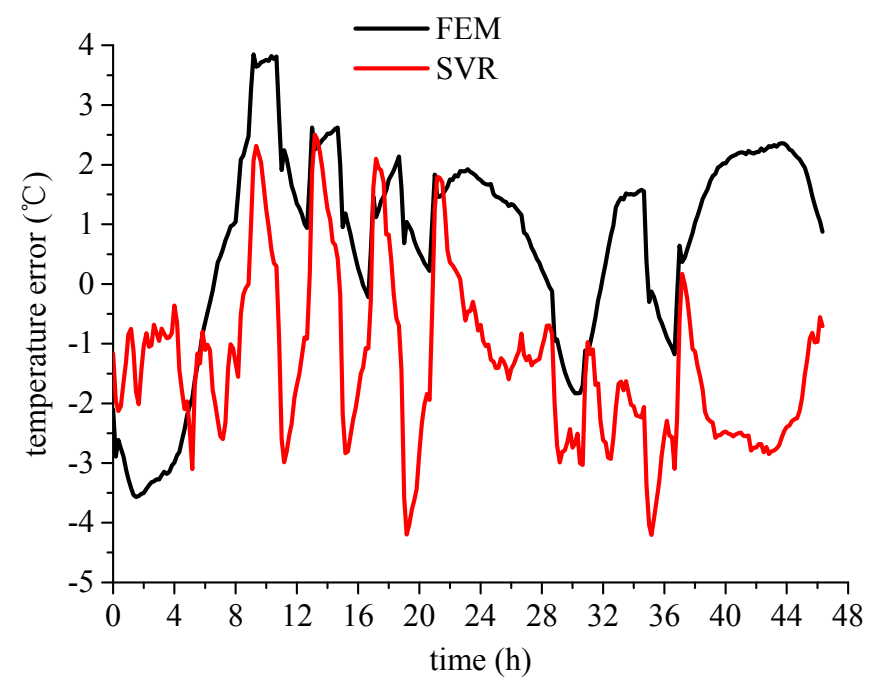

Figure 16. The temperature errors of the proposed method and FEM when $\lambda_{\mathrm{a}}=0.14 \mathrm{~W} /(\mathrm{m} \cdot \mathrm{K})$.

Assuming that the thermal conductivity in thermal analysis $\lambda_{\mathrm{a}}$ of armor wrap was $0.04 \mathrm{~W} /(\mathrm{m} \cdot \mathrm{K})$, the situation is totally different. As seen in Figure 17, the temperature errors of FEM are over $12{ }^{\circ} \mathrm{C}$, much larger than those of SVR. This demonstrates a better generality of the proposed method.

\subsection{The Calculation Speed of the Proposed Method and FEM}

The calculation speeds of SVM and FEM methods were tested on a high performance server. The processor of the server includes two Intel Xeon E5620@2.40 GHz CPUs with total 16 cores and the memory of RAM is 64 GB. The inputs of the proposed model are two surface temperatures and those of FEM are ambient temperature and load currents. The SVM model and FEM model were run in Matlab R2014a and Ansys 12.0, respectively. After training of SVM model and the mesh generation of 3-D FEM model were finished, the solving times with single-step inputs are shown in Table 3. It can be seen that the proposed model is much quicker than the 3-D FEM model and can achieve real-time.

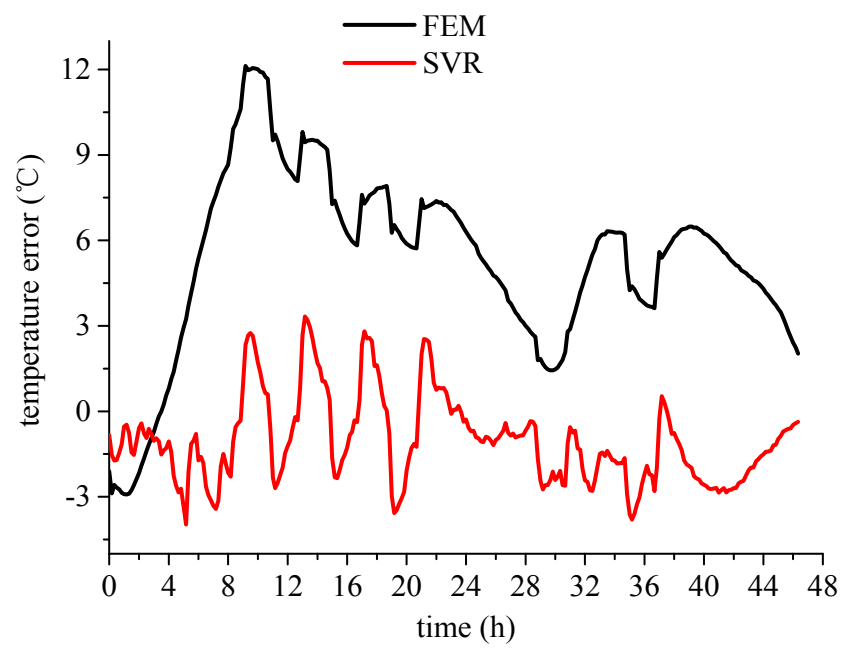

Figure 17. The temperature errors of the proposed method and FEM when $\lambda_{\mathrm{a}}=0.04 \mathrm{~W} /(\mathrm{m} \cdot \mathrm{K})$. 
Table 3. Solving times of single step.

\begin{tabular}{ccc}
\hline Method & SVM Model & 3-D FEM Model \\
\hline Time (s) & 0.03 & 146 \\
\hline
\end{tabular}

Here, some application problems associated with temperature monitoring are discussed. In practical engineering, it is necessary to monitor lots of different cable joints simultaneously. An effective approach for realizing the above function is to collect all the temperature and current information of different joints and then transfer it to the far-end server in real-time. That is to say, one server must calculate numerous temperatures of cable joints in various places. Using the proposed SVM model, the task of the program is to successively put the surface temperature data collected from different cable joints into the SVM model, and then the temperatures inside cable joints will be output soon.

However, the FEM model is totally different because the temperature distribution of cable joint at time $t$ not only depends on the ambient temperature and currents at time $t$, but also relies on temperature information of cable joint at time $t-\Delta t$, where $\Delta t$ is the sampling time. Thus, every time the temperature of cable joint at time $t$ is to be calculated, the solution file at time $t-\Delta t$ should be open as the initial condition of this calculation. This process is called "restart" and the corresponding time $T_{\mathrm{st}}$ is about $43 \mathrm{~s}$. Moreover, running the FEM program consumes lots of memory and CPU resources, which limits the number of the running programs at the same time. Therefore, when lots of cable joints need monitoring, one FEM program must successively perform several thermal analyses of different cable joints during the sampling time. It means that during each sampling time, the database of a joint should be resumed to start calculation and then saved for another calculation in next sampling time. The time of "resume and save" $T_{\mathrm{rs}}$ is nearly $20 \mathrm{~s}$. So, the total time used for one cable joint using FEM $T_{\mathrm{t}} \approx 146+43+20=209 \mathrm{~s}$.

Assume that one server can run three FEM programs at the same time and the sampling time $\Delta t$ is $20 \mathrm{~min}$. According to the above analysis, the 3-D FEM model can only monitor temperatures of 15 different cable joints with uninterrupted calculation using one server while our model can handle about 40,000 cable joints in theory to cover a very large area.

\subsection{Temperature Estimation of Cable Joint under Unbalanced Three-Phase Currents}

The temperature estimation of three-core cable joint studied above was performed under balanced three-phase currents. Nevertheless, in practice, the three-phase currents are often unbalanced, and the three-phase conductor inside cable joint is not all the same. Hence, it is necessary to study the effect of unbalanced currents on the proposed model.

According to [24], the unbalanced degree $\beta$ of three-phase currents is defined as follows,

$$
\beta=\left(I_{\max }-I_{\min }\right) / I_{\max } \times 100 \%
$$

where $I_{\max }$ and $I_{\min }$ are the maximum and minimum of the three-phase currents, respectively. The unbalanced degree $\beta$ should be no more than $25 \%$ in the distribution network.

To analyze the effects of unbalanced three-phase currents, another temperature-rise test of cable joint was carried out under unbalanced currents. The configuration of the test was the same as the previous one. The test lasted $48 \mathrm{~h}$, and the ambient temperature remained nearly $25^{\circ} \mathrm{C}$. The currents were adjusted by changing the number of inducing transformers at each phase and are illustrated in Figure 18 . The maximum unbalanced degree $\beta$ was approximately $55 \%$ to strictly test the performance of the algorithm. 


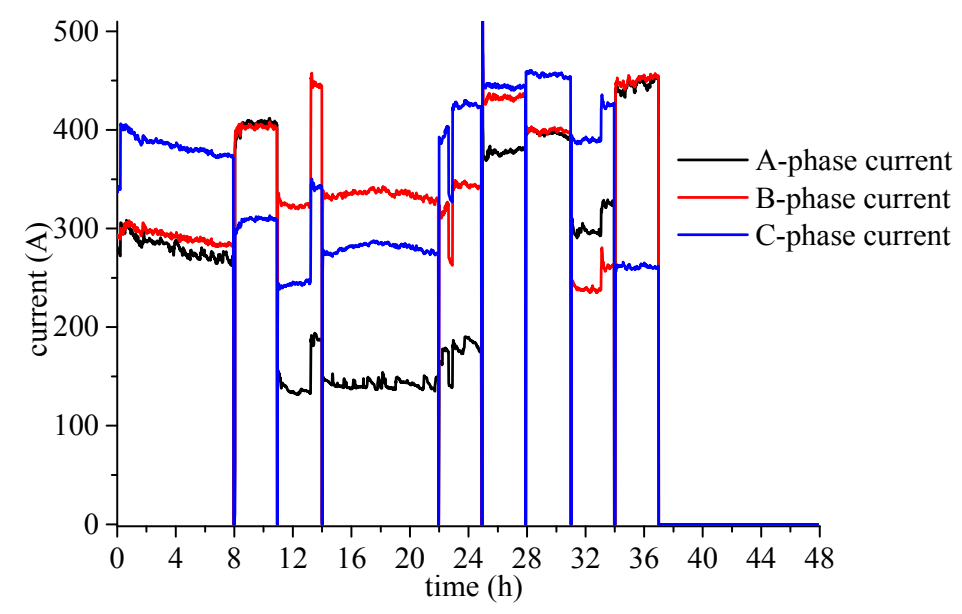

Figure 18. Unbalanced three-phase currents during the temperature-rise test.

Since the inputs of the model are cable surface temperatures only, there is no way to distinguish the temperatures in different phase conductors. Therefore, the model output represents the mean temperature of the three-core cable joint, and if the temperature differences between different conductors are small, this assumption is reasonable. The measured temperatures inside three-phase cable joints and the estimated results are depicted in Figure 19. From the Figure 19a, the estimated temperature based on SVR changes between the maximum and minimum temperatures of the three-core conductor nearly all the time. According to the Figure 19b, the maximum temperature errors of A-phase, B-phase, and C-phase are $6.00{ }^{\circ} \mathrm{C}, 5.03^{\circ} \mathrm{C}$, and $4.44^{\circ} \mathrm{C}$, respectively, which meets the requirement of engineering application. This results demonstrate that our proposed model still has a high precision even under very unbalanced three-phase currents.

\subsection{The Effect of Contact Resistance in Connectors}

As mentioned in the part of Introduction, it was believed in the past that cable joint was not the hot spot in cable system. As seen in the literatures [16,17], the temperature inside joints was calculated cooler than that of cable. The reason given by those papers was that the ferrule diameter was considerably larger than that of the conductor $(27.2 \mathrm{~mm}$ compared with $19.1 \mathrm{~mm})$ without consideration of contact resistance. But in fact, the contact resistance constitutes a non-negligible proportion of the whole resistance of connector. According to our measurement result, the measured total resistance in $G_{2}$, as shown in Figure 20, is about $7.5 \mu \Omega$. In the contrast, the resistance ignoring contact effect $R^{\prime}=\rho \times l_{2} /\left(\pi\left(r_{1}+r\right)^{2}\right)$. Since $\rho=1.75 \times 10^{-8} \Omega \cdot \mathrm{m}, l_{2}=0.12 \mathrm{~m}, r=10.25 \mathrm{~mm}, r_{1}=6.1$ $\mathrm{mm}, R^{\prime}$ can be calculated to be $2.5 \mu \Omega$, which is only one-third of the measured value. What's more, the experimental results recorded in [2,3] also demonstrated that the joint is indeed the hot spot in a three-core cable. Therefore, it is necessary to pay close attention to the contact resistance, of which the varying range is a key parameter.

As for the range of equivalent resistance under incorrect contacting conditions, there are two different situations in our opinions.

The first case is lack of pressure. As is widely known, the equivalent resistance of the connector depends upon contact surface and pressure. If the pressure is not enough, the contact resistance will be larger. But it is really difficult to quantify the range of contact resistance because the minimum pressure is hard to determine. In the most extreme case, the connector just contacts with the conductor and there is no external pressure applied to the interface, the equivalent resistance might be over several Ohms. 


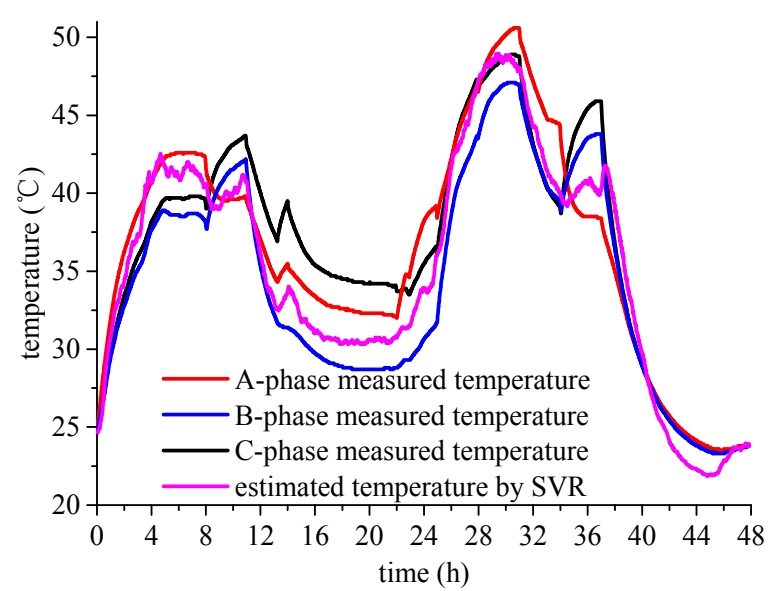

(a)

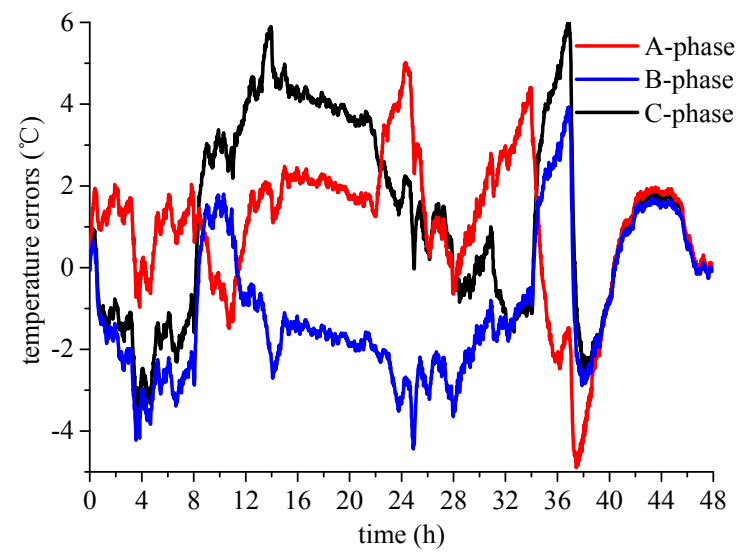

(b)

Figure 19. The measured results and estimated temperature based on SVR under unbalanced three-phase currents. (a) Measured and estimated temperatures; (b) Temperature errors.

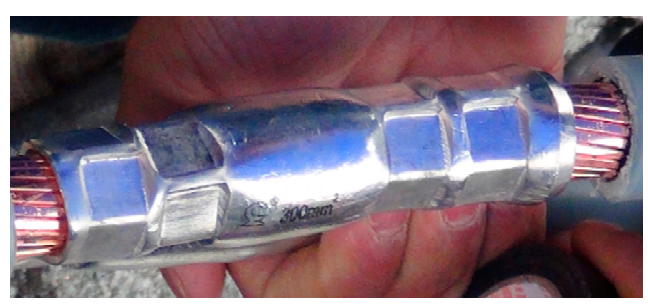

Figure 20. Connectors pressed with four indentations.

Another incorrect contacting condition is not enough indentations. As shown in Figure 20, there are four indentations in one connector according to the rule of manufacturer and each indentation corresponds to a local contact resistance. In the above experiment, four local contact resistances with parallel connection are nearly $7.5 \mu \Omega$ so that one local contact resistance is about $30 \mu \Omega$. If a worker presses the connector with only one indentations (it is generally impossible), the total equivalent resistance will be $30 \mu \Omega$, which is the possible maximum contact resistance due to insufficient indentations.

Based on our aforementioned analysis, there is no typical range of equivalent resistance under incorrect contacting conditions, mainly because of too large range of pressure. Under normal condition, however, we suppose that there is a typical range of resistance related to the application. Normal 
condition means that both the pressure and the number of indentations are in accordance with the rule of manufacture under the assumption that there is little difference between various manufacturers. The number of indentations are usually constant but the pressure changes over the range of 40-60 MPa according to the experience of the worker who installed the test joint. Assuming that the equivalent resistance is nearly linear with the pressure, the equivalent resistance can vary about $50 \%$. For more accurate analysis, it requires further research and we will measure the equivalent resistance with different pressures and conductor cross-sections in our next study.

\section{Conclusions}

The paper put forward an approach to estimate the temperature inside the three-core cable joint. The model was established by SVR with two fixed cable surface temperatures as inputs, and the effectiveness of this algorithm was validated by the temperature-rise test. The results are summarized as follows.

(1) Through parameter optimization, it is recommended that model needs two measuring spots in the cable, with one spot adjacent to the joint and the other one remote enough from the joint for axial heat flux to be zero.

(2) The temperature-rise test of a $15 \mathrm{kV}$ three-core cable joint under nearly balanced three-phase currents was carried out. The estimated temperature is in good agreement with the measured one with the maximum error of approximately $4{ }^{\circ} \mathrm{C}$.

(3) Although the thermal conductivity of armor wrap used for thermal analysis was different from its real value, the temperature could still be estimated accurately based on SVR. This indicates that the proposed model can effectively overcome the influence of uncertainty of thermal conductivity in armor wrap.

(4) The accuracies of the proposed model was compared with those of FEM, showing a better generality.

(5) The proposed model is much quicker than FEM with the solving time of about $0.03 \mathrm{~s}$ in single step and can achieve real-time.

(6) A temperature-rise test under quite unbalanced three-phase currents was performed. Compared with the measured results, the maximum estimated error based on our model is no more than $6{ }^{\circ} \mathrm{C}$. The results indicate that this model can accurately obtain the real-time temperature of three-cable joint no matter how the currents change.

Author Contributions: Conceptualization, J.R. and Q.Z.; Data curation, L.T. and K.T.; Formal analysis, L.T.; Writing original draft, L.T.

Conflicts of Interest: The authors declare no conflicts of interest.

\section{References}

1. Wang, H.L.; Zheng, S.J.; Chen, L. The statistical analysis of power cable fault in Jiangxi power grid. Jiangxi Electr. Power 2013, 37, 55-57.

2. Yang, F.; Liu, K.; Cheng, P.; Wang, S.; Wang, X.Y.; Gao, B.; Fang, Y.L.; Xia, R.; Ullah, I. The coupling fields characteristics of cable joints and application in the evaluation of crimping process defects. Energies 2016, 9, 932. [CrossRef]

3. Wang, P.Y.; Liu, G.; Ma, H.; Liu, Y.G.; Xu, T. Investigation of the ampacity of a prefabricated straight-through joint of high voltage cable. Energies 2017, 10, 2050. [CrossRef]

4. Das, S.; Majumder, R.; Singh, S. Cable joint installation time optimization. IEEE Trans. Dielectr. Electr. Insul. 2017, 24, 3959-3965. [CrossRef]

5. Gouda, O.E.; El, D.A.Z. Electrothermal analysis of low- and medium-voltage cable joints. Electr. Power Compon. Syst. 2016, 44, 110-121. [CrossRef]

6. Wang, P. Temperature Calculation and Experimental Study of $10 \mathrm{kV}$ Three-Core Cable and Accessories. Master's Thesis, South China University of Technology, Guangzhou, China, 2016. 
7. Church, E.A. Temperature in electric power cables under variable loading. Trans. Am. Inst. Electr. Eng. 1931, 50, 982-991. [CrossRef]

8. Wollaston, F.O. Transient temperature phenomena of 3-conductor cables. Trans. Am. Inst. Elect. Eng. 1949, 68, 1284-1297. [CrossRef]

9. Lyall, J. Two dimensional modeling of three core cable transient temperature rise. IEEE Trans. Power Deliv. 1990, 5, 21-25. [CrossRef]

10. Anders, G.J.; Napieralski, A.K.; Zamojski, W. Calculation of the internal thermal resistance and ampacity of 3-core unscreened cables with fillers. IEEE Trans. Power Deliv. 1998, 13, 699-705. [CrossRef]

11. Anders, G.J.; Napieralski, A.K.; Kulesza, Z. Calculation of the internal thermal resistance and ampacity of 3-core screened cables with fillers. IEEE Trans. Power Deliv. 1999, 14, 729-734. [CrossRef]

12. Haiqing, N.; Jia, Y.; Kaifa, Y. Calculation of transient cable conductor temperature based on support vector machine optimized by particle swarm optimization algorithm. In Proceedings of the IEEE International Conference on High Voltage Engineering and Application, Chengdu, China, 19-22 September 2016.

13. Bragatto, T.; Cresta, M.; Gatta, F.M.; Geri, A.; Maccioni, M.; Paulucci, M. Determination method of current-carrying capacity of HVAC cable joint based on ANSYS. Electr. Power Syst Res. 2017, 149, 190-197. [CrossRef]

14. Liu, G.; Wang, P.Y.; Wang, Z.H.; Xu, T.; Liu, Y.G.; Han, Z.Z. Underground MV power cable joints: A nonlinear thermal circuit model and its experimental validation. J. South China Univ. Technol. (Nat. Sci. Ed.) 2017, 45, 22-28.

15. Liu, G.; Wang, Z.H.; Xu, T.; Liu, Y.G.; Wang, P.Y. Current capacity calculation and experimental analysis of $110 \mathrm{kV}$ cable joint. High Volt. Eng. 2017, 43, 1670-1676.

16. Weedy, B.M. Prediction of steady state temperatures in a three-core distribution cable and splice. Electr. Power Syst. Res. 1986, 10, 1-5. [CrossRef]

17. Weedy, B.M. Prediction of the temperatures in a three-core distribution cable and splice with load changes. Electr. Power Syst. Res. 1987, 13, 43-48. [CrossRef]

18. Xu, Y.Z.; Wang, L.T.; Gao, G.X.; Xie, X.Z. The oretical study on temperature field distribution of power cable junction. Power Syst. Prot. Control 2008, 36, 4-7.

19. Qiu, Z.B.; Ruan, J.J.; Huang, D.C.; Pu, Z.H.; Shu, S.W. A prediction method for breakdown voltage of typical air gaps based on electric field features and support vector machine. IEEE Trans. Dielectr. Electr. Insul. 2015, 22, 2125-2135. [CrossRef]

20. IEC 60751. Industrial Platinum Resistance Thermometers and Platinum Temperature Sensors; International Electrotechnical Commission: Geneva, Switzerland, 2016.

21. IEC 60853. Calculation of the Cyclic and Emergency Rating of Cables, Part 2; International Electrotechnical Commission: Geneva, Switzerland, 1989.

22. X, Z.J. Heat Transfer; China Machine Press: Beijing, China, 1980.

23. IEC 60287. Electric Cables-Calculation of the Current Ratings, Part 2-1; International Electrotechnical Commission: Geneva, Switzerland, 1989.

24. Q/GDW 1519. Operation and Maintenance Regulation of Distribution Network; State Grid Corporation of China: Beijing, China, 2014.

(C) 2018 by the authors. Licensee MDPI, Basel, Switzerland. This article is an open access article distributed under the terms and conditions of the Creative Commons Attribution (CC BY) license (http://creativecommons.org/licenses/by/4.0/). 